TACKY'S REVOLT 



\title{
TACKY'S REVOLT
}

\author{
The Story of an \\ Atlantic Slave War
}

\section{VINCENT BROWN}

THE BELKNAP PRESS OF

HARVARD UNIVERSITY PRESS

Cambridge, Massachusetts

London, England

2020 


\section{Copyright $(9) 2020$ by Vincent Brown \\ All rights reserved \\ Printed in the United States of America}

\section{First printing}

Brown jacket credits

Jacket illustration by Robert Fujitani, from The Story of Jamaica (Kingston, I962), The Pioneer Press. () The Gleaner Company (Media) Limited.

$$
\begin{gathered}
\text { Jacket design by Lisa Roberts } \\
9780674242098 \text { (EPUB) } \\
9780674242 \text { I04 (MOBI) } \\
978067424208 \text { I (PDF) }
\end{gathered}
$$

The Library of Congress has cataloged the printed edition as follows:

Names: Brown, Vincent, I967- author.

Title: Tacky's revolt : the story of an Atlantic slave war / Vincent Brown.

Description: Cambridge, Massachusetts : The Belknap Press of

Harvard University Press, 2020. I Includes bibliographical references and index.

Identifiers: LCCN 2019028500 I ISBN 9780674737570 (alk. paper)

Subjects: LCSH: Slave insurrections-Jamaica-History— I 8th century. I

Slavery-Jamaica-History-I8th century. I Great Britain-Colonies-America.

Classification: LCC HTio96 .S 2019 I DDC 306.3 / 62097292-dc23

LC record available at https://lccn.loc.gov/20I 9028500 